\title{
Template-Free Synthesis and Characterization of Single-Phase Voided Poly(o-anisidine) and Polyaniline Colloidal Spheres
}

\author{
Yiwei Tan, Feng Bai, Dingsheng Wang, Qing Peng, Xun Wang, Yadong Li*
}

Department of Chemistry, Tsinghua University, Beijing 100084, China
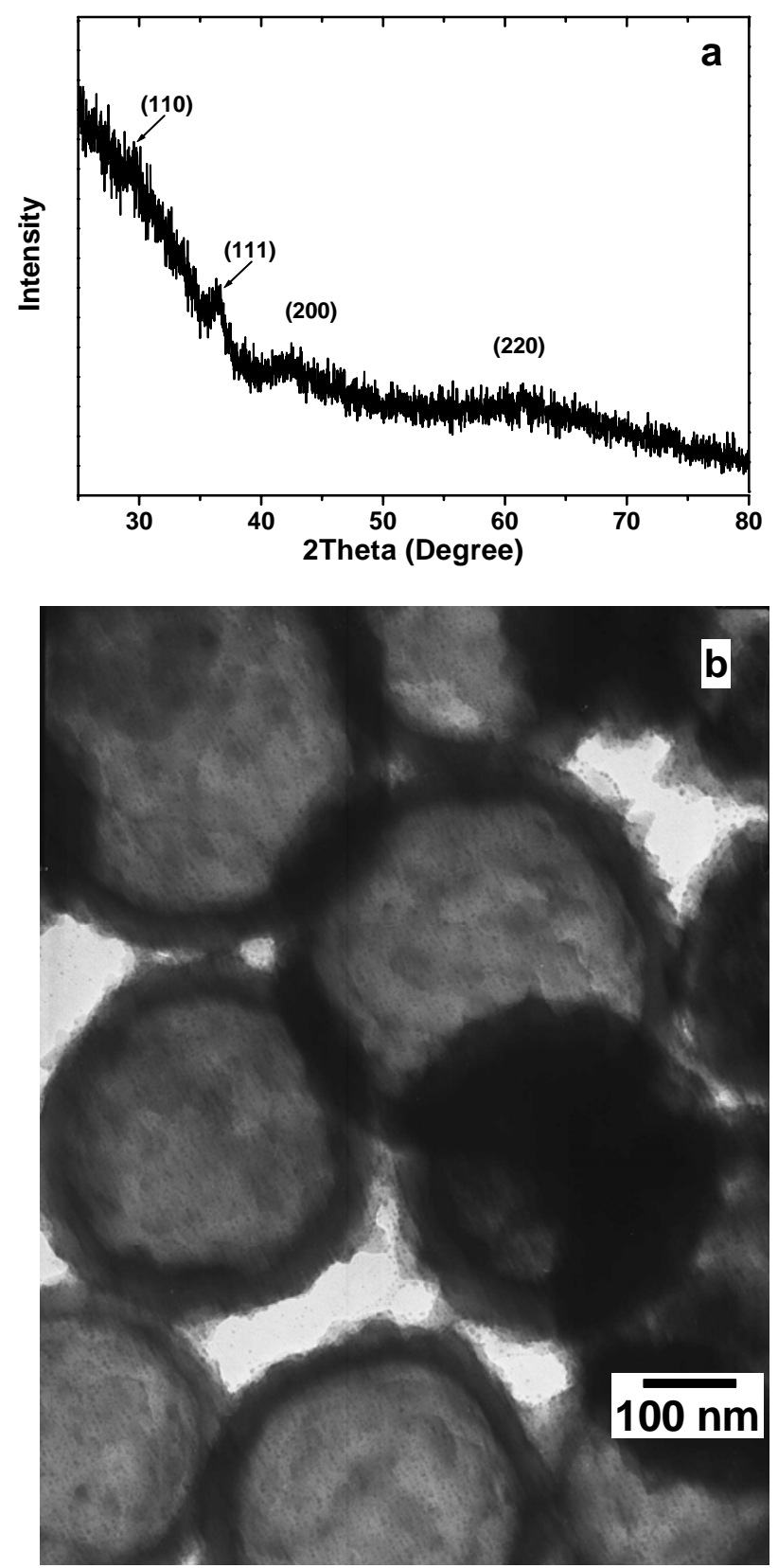

Figure S1. (a) XRD pattern of cuprite $\mathrm{Cu}_{2} \mathrm{O}$ nanoparticles and (b) TEM image of $\mathrm{Cu}_{2} \mathrm{O}$ nanoparticles wrapped in hollow poly $(o$-anisidine) particles. The sample for XRD measurements was prepared by repeatedly casting the resulting aqueous solution on a clean glass slide and drying naturally. X-ray diffraction measurements were performed with $\mathrm{Cu} k \alpha(1.54 \AA)$ radiation at $40 \mathrm{kV}$ and $200 \mathrm{~mA}$. The specimen for TEM examination was prepared by depositing a drop of the resulting aqueous solution on a carbon-coated copper grid and allowing water to evaporate at room temperature.

To identify the reduced product of $\mathrm{Cu}^{2+}$ ions, X-ray diffraction (XRD) pattern is recorded from a sample obtained at $180{ }^{\circ} \mathrm{C}$ over $7 \mathrm{~h}$ of the hydrothermal reaction between $0.02 \mathrm{M} o$-anisidine and cupric bromide, basic in the presence of cetyltrimethylammonium bromide 
(CTAB) (Method II). As shown in Figure S1a, these typical diffraction features are in accordance with the crystal data of cubic cuprite structure. Broad peaks are characteristic of colloidal crystal clusters owing to the very small size of the particles. The mean crystalline size of the as-synthesized cuprous oxide nanoparticles is calculated to be $3.5 \mathrm{~nm}$ by measuring the peak width of the X-ray diffraction lines according to the Debye-Scherrer equation $(D=0.9 \lambda / \beta \cos \theta)$. The corresponding TEM image reveals that small $\mathrm{Cu}_{2} \mathrm{O}$ nanoparticles are entrapped on hollow poly $(o$-anisidine) particles (Figure $\mathrm{S} 1 \mathrm{~b})$. The average size of the as-prepared $\mathrm{Cu}_{2} \mathrm{O}$ nanoparticles is $3.1 \mathrm{~nm}$, which is determined by arbitrarily measuring the diameters of more than 150 individual particles. This is in good agreement with the value (3.5 nm) obtained according to the XRD pattern. Small $\mathrm{Cu}_{2} \mathrm{O}$ nanoparticles can be separated from the hollow poly $(o$-anisidine $)$ colloids after centrifugation at $4500 \mathrm{rpm}$ for $5 \mathrm{~min}$. In the case of Method $\mathrm{I}$ (using $\mathrm{Cu}(\mathrm{Ac})_{2}$ as the initiator), the reduced product of $\mathrm{Cu}^{2+}$ ions is also $\mathrm{Cu}_{2} \mathrm{O}$, while the SEM and TEM studies show the nanowire structure. The detailed studies will be reported separately.

The overall oxidative polymerization reaction of $o$-anisidine initiated by $\mathrm{Cu}^{2+}$ can be described by the equation illustrated in Scheme 1 .

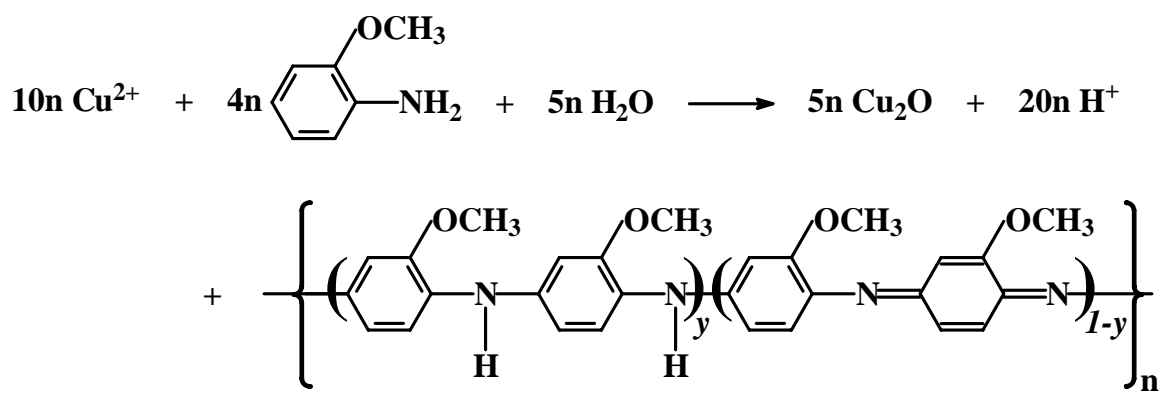

Scheme 1. The reaction equation of $o$-anisidine polymerization initiated by $\mathrm{Cu}^{2+}$ ions in an aqueous solution.

The reaction between $\mathrm{Cu}(\mathrm{Ac})_{2}$ and cationic surfactant $\mathrm{CTAB}$ yields green precipitates of cupric bromide, basic $\left(\mathrm{Cu}_{2}(\mathrm{OH})_{3} \mathrm{Br}\right)$ that can be isolated. Figure S2 shows the X-ray powder diffraction pattern of the green precipitates. The peaks can be perfectly indexed to crystalline cupric bromide, basic $\left(\mathrm{Cu}_{2}(\mathrm{OH})_{3} \mathrm{Br}\right.$, JCPDF no. 74-1652). Note that cupric bromide, basic has another phase $\left(\mathrm{CuBr}_{2}\left(\mathrm{Cu}(\mathrm{OH})_{2}\right)_{3}, \mathrm{JCPDF}\right.$ no. 72-1279). Here, we tentatively assign the peaks according to the $\mathrm{Cu}_{2}(\mathrm{OH})_{3} \mathrm{Br}$ phase.

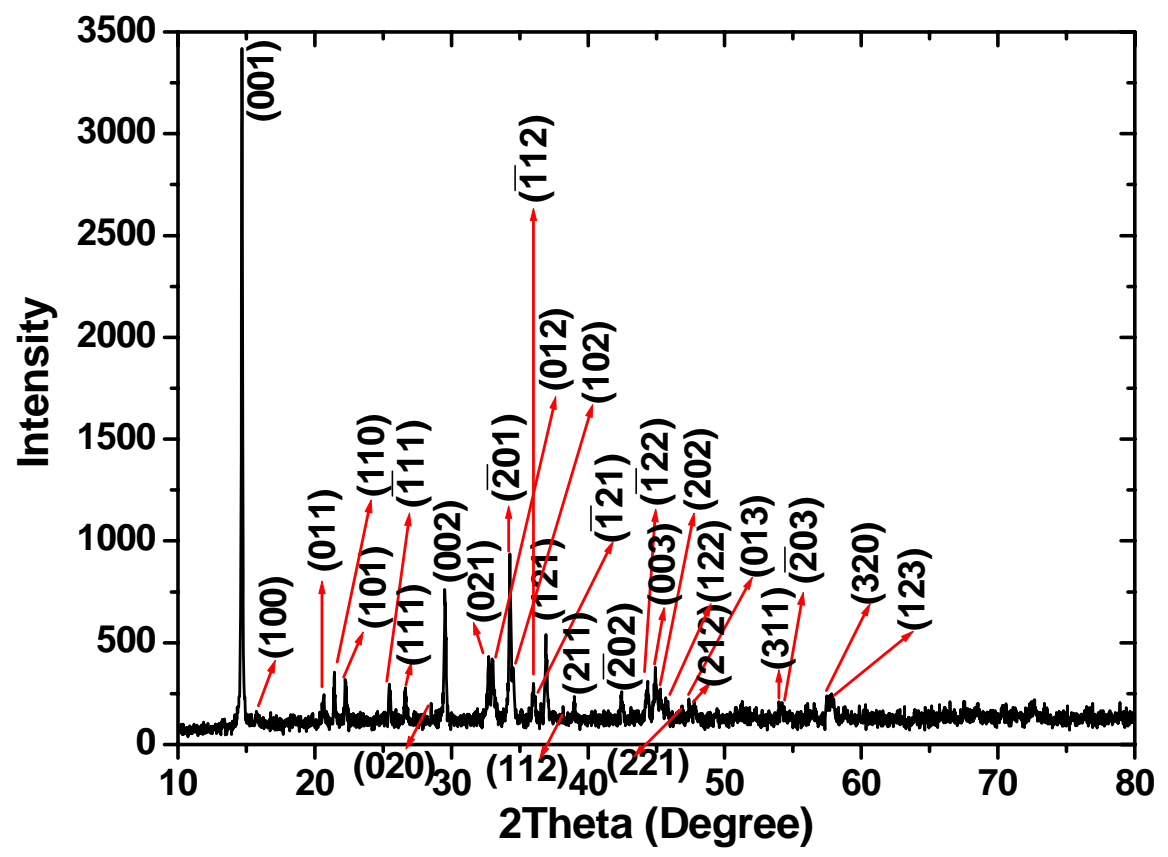

Figure S2. X-ray powder diffraction pattern of cupric bromide, basic obtained by the reaction of $\mathrm{Cu}(\mathrm{Ac})_{2}$ with $\mathrm{CTAB}$. 